\title{
Article \\ Chlorin Endogenous to the North Pacific Brittle Star Ophiura sarsii for Photodynamic Therapy Applications in Breast Cancer and Glioblastoma Models
}

\author{
Antonina Klimenko ${ }^{1,+}$, Elvira E. Rodina ${ }^{1,+}$, Denis Silachev ${ }^{2,+}$, Maria Begun ${ }^{1}$, Valentina A. Babenko ${ }^{2}$, \\ Anton S. Benditkis ${ }^{3}$, Anton S. Kozlov ${ }^{3}$, Alexander A. Krasnovsky ${ }^{3}$, Yuri S. Khotimchenko ${ }^{1}$ \\ and Vladimir L. Katanaev $1,4, *$ (D)
}

check for updates

Citation: Klimenko, A.; Rodina, E.E.; Silachev, D.; Begun, M.; Babenko, V.A.; Benditkis, A.S.; Kozlov, A.S.; Krasnovsky, A.A.; Khotimchenko, Y.S.; Katanaev, V.L. Chlorin Endogenous to the North Pacific Brittle Star Ophiura sarsii for Photodynamic Therapy Applications in Breast Cancer and Glioblastoma Models. Biomedicines 2022, 10, 134. https://doi.org/10.3390/

biomedicines 10010134

Academic Editors: Kyungsu Kang and Stefano Bacci

Received: 2 December 2021

Accepted: 5 January 2022

Published: 8 January 2022

Publisher's Note: MDPI stays neutral with regard to jurisdictional claims in published maps and institutional affiliations.

Copyright: (C) 2022 by the authors. Licensee MDPI, Basel, Switzerland. This article is an open access article distributed under the terms and conditions of the Creative Commons Attribution (CC BY) license (https:// creativecommons.org/licenses/by/ $4.0 /)$
1 Institute of Life Sciences and Biomedicine, Far Eastern Federal University, 690922 Vladivostok, Russia; klimenko.am@dvfu.ru (A.K.); rodina.ee@dvfu.ru (E.E.R.); begun.ma@dvfu.ru (M.B.); khotimchenko.ys@dvfu.ru (Y.S.K.)

2 A.N. Belozersky Research Institute of Physico-Chemical Biology, Moscow State University, 119899 Moscow, Russia; proteins@mail.ru (D.S.); babenkova@belozersky.msu.ru (V.A.B.)

3 Federal Research Center of Biotechnology of the Russian Academy of Sciences, 119071 Moscow, Russia; anton93benditkis@yandex.ru (A.S.B.); anton4ikk_06@mail.ru (A.S.K.); phoal@mail.ru (A.A.K.)

4 Translational Research Center in Oncohaematology, Department of Cell Physiology and Metabolism, Faculty of Medicine, University of Geneva, 1211 Geneva, Switzerland

* Correspondence: vladimir.katanaev@unige.ch

+ These authors contributed equally to this work.

Abstract: Photodynamic therapy (PDT) represents a powerful avenue for anticancer treatment. PDT relies on the use of photosensitizers-compounds accumulating in the tumor and converted from benign to cytotoxic upon targeted photoactivation. We here describe $(3 S, 4 S)$-14-Ethyl-9(hydroxymethyl)-4,8,13,18-tetramethyl-20-oxo-3-phorbinepropanoic acid (ETPA) as a major metabolite of the North Pacific brittle stars Ophiura sarsii. As a chlorin, ETPA efficiently produces singlet oxygen upon red-light photoactivation and exerts powerful sub-micromolar phototoxicity against a panel of cancer cell lines in vitro. In a mouse model of glioblastoma, intravenous ETPA injection combined with targeted red laser irradiation induced strong necrotic ablation of the brain tumor. Along with the straightforward ETPA purification protocol and abundance of $O$. sarsii, these studies pave the way for the development of ETPA as a novel natural product-based photodynamic therapeutic.

Keywords: porphyrin; chlorin; singlet oxygen; photodynamic therapy; ophiura; cancer; breast cancer; glioblastoma; mouse models

\section{Introduction}

Photodynamic therapy (PDT) relies on the use of photosensitizers-compounds that are relatively benign until excited by light of a particular wavelength that converts them into an activated state resulting in the generation of reactive oxygen species [1-3]. PDT finds multiple applications in medicine, particularly in anticancer therapy, where it has been approved to treat cancers in the skin (basal cell carcinoma), lungs, or esophagus [2,3]. Other forms of cancer, such as in the breast or brain (e.g., glioblastoma) have so far evaded approved PDT applications [2,3].

A number of photosensitizers have been marketed for PDT in different countries, and the search for novel compounds never ceases [3,4]. Photosensitizers activatable in the red part of the spectrum are particularly sought, as the red light can penetrate deeper in biological tissues [1,3]. Derived from chemical synthesis, these complex compounds weigh upon the costs of PDT; stability in body fluids is another issue with synthetic photosensitizers $[3,4]$. 
Natural products have always been one of the major sources of new drugs, including oncology therapeutics [5-7]. Porphyrin-type compounds such as chlorins can act as efficient photosensitizers and have been found in diverse living groups $[4,8]$. In a search for novel anticancer compounds from ophiuras [9], we have recently discovered the chlorin $(3 S, 4 S)$ 14-Ethyl-9-(hydroxymethyl)-4,8,13,18-tetramethyl-20-oxo-3-phorbinepropanoic acid (ETPA) from a North Pacific brittle star Ophiura sarsii-the first-ever porphyrin identified in Ophiuroidea (phylum Echinodermata) [10]. This discovery was unexpected, as Ophiuroidea were believed to lack porphyrin/chlorin synthesis [11] and raised the hypothesis that the Ophiura sarsii chlorin was the result of dietary (and perhaps seasonal) consumption by these marine invertebrates [10].

In the current work, we provide evidence that ETPA production is endogenous to Ophiura sarsii and is independent of their food consumption. As a major metabolite in this abundant brittle star species and amenable to simple purification, the $663 \mathrm{~nm}$ lightabsorbing chlorin shows sub-micromolar phototoxicity against a panel of cancer cells in vitro and serves as an efficient PDT against glioblastoma in a mouse model. Our findings pave the way for the development of ETPA as a natural photosensitizer in a broad spectrum of PDT applications.

\section{Materials and Methods}

\subsection{Species Collection and Food Deprivation}

Brittle stars Ophiura sarsii were collected at the depths of 15-18 m near the Vyatlin Cape (Russky Island) in the Peter the Great Gulf, Sea of Japan, in December 2020. Sample collection was performed by the standards approved by the Ministry of Science and Higher Education (Russia); all efforts were made to minimize animal suffering. Brittle stars (wet weight $-25 \mathrm{~g}$ ) were separated into two groups. The first group, after thorough $2 \times$ running water rinsing, was frozen and stored at $-80^{\circ} \mathrm{C}$. The second was placed into a clean spacious aquarium regularly refilled with fresh sterilized and filtered seawater without any source of food. Gradual emptying of the animals' digestive tracks could be visually seen in the first days of such fasting. After 12 days, the food-deprived animals were rinsed and stored as the first group.

\subsection{Homogenization and Extraction}

O. sarsii were mechanically homogenized and then sequentially placed in solvents with increasing polarity: hexane, chloroform, ethanol, and water $(125 \mathrm{~mL}$ each) at room temperature for 8-12 h, with constant stirring on a PSU-10i shaker (Biosan, Riga, Latvia). The ratio of solvents to the homogenized mass was 5:1 (volume). The resulting extracts were cleared through paper filters and concentrated on a Hei-VAP Value Rotary Evaporator (Heidolph, Schwabach, Germany) at $37-40{ }^{\circ} \mathrm{C}$ under vacuum (chemical vacuum station PC 3002 VARIO (Vacuubrand, Wertheim, Germany)). The chlorin (3S,4S)-14-Ethyl-9(hydroxymethyl)-4,8,13,18-tetramethyl-20-oxo-3-phorbinepropanoic acid (ETPA) localized to the ethanol fraction [10] that contained $721.5 \mathrm{mg}$ (control brittle stars) and $579 \mathrm{mg}$ (fasted brittle stars) dry weight. To remove salts from the ethanol extract, liquid-liquid extraction was performed. The ethanol extract was dissolved in $30 \mathrm{~mL} \mathrm{n}$-butanol and $50 \mathrm{~mL}$ water and transferred to a separatory funnel. The organic (butanol) phase was extracted $3 \times$ with $50 \mathrm{~mL}$ water. Then, $\mathrm{n}$-butanol was removed from the fraction using the rotary evaporator, followed by dissolution of the precipitate in methanol for subsequent chromatography.

\subsection{Analytical and Semi-Preparative HPLC}

Analytical and Semi-Preparative HPLC was performed on a Shimadzu system (Shimadzu, Kyoto, Japan) equipped with LC-20AP modular pumps, an SPD-20A spectrophotometric detector, and an FRC-10A fraction collector. Analytical separation was performed on a Shim-pack SHIMADZU GIST C18 column $(250 \mathrm{~mm} \times 4.6 \mathrm{~mm}$, particle size $5 \mu \mathrm{m})$. $\mathrm{MeOH}$ was used as the mobile phase solution $\mathrm{A}$, and water acidified with $0.1 \%$ formic acid-as solution B. Chromatography was performed at a rate of $0.6 \mathrm{~mL} / \mathrm{min}$ at $40^{\circ} \mathrm{C}$, 
with a maximum pressure of $10 \mathrm{MPa}$. Elution was achieved with a gradient: from 50 to $95 \% \mathrm{MeOH}$ solution in $55 \mathrm{~min}$, then $95 \%$ solution for $10 \mathrm{~min}$, then returned to $50 \% \mathrm{MeOH}$ in $5 \mathrm{~min}$. Reanalysis was performed after complete conditioning of the column. Detection was carried out on a spectrophotometric detector at wavelengths of $210 \mathrm{~nm}$ and $366 \mathrm{~nm}$.

Separation of extracts was carried out on a Shim-pack SHIMADZU GIST C18 column $(250 \mathrm{~mm} \times 10.00 \mathrm{~mm}$, particle size $5 \mu \mathrm{m}$ ) with a mobile phase of methanol (solution A) and water acidified with $0.1 \%$ formic acid (solution B). Chromatography was performed at a rate of $2.6 \mathrm{~mL} / \mathrm{min}$ at $23{ }^{\circ} \mathrm{C}$. The gradient was from 80 to $97 \%$ solution in $50 \mathrm{~min}, 97 \%$ solution $\mathrm{A}$ in $8 \mathrm{~min}$, then return to $80 \% \mathrm{MeOH}$ in $7 \mathrm{~min}$ with $\mathrm{UV}$ detection at $210 \mathrm{~nm}$ and $366 \mathrm{~nm}$.

\subsection{High-Resolution Spectrophotometry}

High-Resolution Spectrophotometry of the chromatographic fraction containing ETPA dissolved in methanol was performed by a UV-1800 spectrophotometer (Shimadzu, Kyoto, Japan) in the wavelength range from 200 to $800 \mathrm{~nm}$.

\subsection{Cells and Medium}

Human breast cancer cells MCF-7, BT-20 and MDA-MB-231 (all from ATCC, atcc.org), rat C6 glioma cells and human embryonic kidney (HEK-293 cells, both from Collection of vertebrate cell cultures, Institute of Cytology, Russian Academy of Sciences (incras.ru/wpcontent/uploads/2019/06/katalog_rccc_v_2018_rus.pdf, accessed on 21 December 2021) were cultured in DMEM + GlutaMAX medium (Gibco, Waltham, MA, USA) supplemented with 10\% fetal bovine serum (Biosera, Nuaille, France) and 1\% antibiotic-antimycotic (Gibco, USA). Cell cultures were grown in a $\mathrm{CO}_{2}$ incubator with a Galaxy 48R cell vitality monitoring and vitality system (Eppendorf, Hamburg, Germany) at $37^{\circ} \mathrm{C}$ and $5 \% \mathrm{CO}_{2}$.

\subsection{Photoxicity Assays}

ETPA was dissolved in DMSO. In 96-well microculture plates, human breast cancer and glioma cells were seeded at the density of 3000 cells/well and cultured overnight in a $\mathrm{CO}_{2}$ incubator at $37^{\circ} \mathrm{C}$. After medium removal, $50 \mu \mathrm{L}$ DPBS with serial dilutions of ETPA was added for $2 \mathrm{~h}$ (the resultant concentration of DMSO in the wells was $<0.2 \%$ ). Cells in pure DPBS served as a positive control; wells with DPBS without cells served as a negative control. Next, the cells were irradiated with red light in the wavelength range from 580 to $780 \mathrm{~nm}$ using a 2000-4000 lux LED lamp for $30 \mathrm{~min}$. Removal of ETPA immediately before light exposure did not reduce/influence the resulting phototoxicity. Photosynthetic photon flux density (PPFD) was measured in 12 randomly chosen wells using the LI190R Quantum Sensor (LI-COR Biosciences, Lincoln, NE, USA) and found to vary from $217-580 \mu \mathrm{mol} / \mathrm{m}^{2} / \mathrm{s}$. Fluence was measured (using a Newport Optical Power Meter 842PE, MKS Instruments, Norwood, MA, USA) to be $16.04 \mathrm{~J} / \mathrm{cm}^{2}$. Next, a $200 \mu \mathrm{L}$ culture medium was added to each well for additional incubation for $72 \mathrm{~h}$ in a $\mathrm{CO}_{2}$ incubator at $37^{\circ} \mathrm{C}$.

To assess cell death, the culture medium was removed and $50 \mu \mathrm{L}$ of MTT (triazolyl blue tetrazolium bromide) reagent (DIA-M, Moscow, Russia) dissolved in DPBS at $0.5 \mathrm{mg} / \mathrm{mL}$ was added for $3 \mathrm{~h}$ incubation at $37^{\circ} \mathrm{C}$. After aspiration of the liquid, $100 \mu \mathrm{L}$ DMSO was added to each well for $5 \mathrm{~min}$ before measuring the optical density at wavelengths of 570 and $630 \mathrm{~nm}$ using a Cytation 5 multifunctional plate reader (BioTek, Winooski, VT, USA). MTT was separately performed for C6 and HEK-293 cells in the dark to obtain light-independent cytotoxicity. $\mathrm{IC}_{50}$ and standard error of the mean (SEM) were obtained by standard dose-response curve fitting using GraphPad Prism 8.

\subsection{Determination of the Singlet Oxygen Quantum Yield of ETPA}

Singlet oxygen was detected using two methods. One of them was based on the measurement of the rates of chemical trapping of singlet oxygen by 1,3-diphenylisobenzofuran (DPIBF) (Acros Organics, Geel, Belgium, >99\%) as described [12,13]; see Supplementary 
Figure S1. Having a strong absorption maximum at $414 \mathrm{~nm}$, DPIBF is efficiently oxidized by singlet oxygen forming colorless products having no absorption maxima in the visible spectral region. The rate of DPIBF bleaching is directly proportional to the rate of ${ }^{1} \mathrm{O}_{2}$ production by irradiation of a photosensitizer. The absorption spectra of the trap solutions were recorded with the SF-56 spectrophotometer (LOMO Spektr, St. Petersburg, Russia). For irradiation, a xenon lamp and grating monochromator were employed.

Another method was based on detection of the infrared phosphorescence of singlet oxygen at $1270 \mathrm{~nm}$, which arises due to the energy transfer from the triplet state of the photosensitizer molecules to oxygen, followed by the population of singlet oxygen (the reactive excited singlet $\left({ }^{1} \Delta_{g}\right)$ state of oxygen molecules); see Supplementary Figure S2. Measurements were carried out using a laser/LED spectrometer assembled at the Federal Research Center of Biotechnology of the Russian Academy of Sciences [14]. The spectrometer allowed phosphorescence detection upon excitation by pulses of LED with the emission maxima at $399 \mathrm{~nm}$ (Polironik, Moscow, Russia). Phosphorescence was recorded at a $90^{\circ}$ angle with respect to the excitation beam through the cut-off filter that transmitted IR light at $\lambda>1000 \mathrm{~nm}$ and one of three interchangeable interference filters with transmission maxima at 1230, 1270, and $1310 \mathrm{~nm}$ and half-width of $10 \mathrm{~nm}$. The photodetector was an FEU-112 photomultiplier (Ekran Optical Systems, Novosibirsk, Russia) (PMT), with the S-1 spectral response cooled to $-35^{\circ} \mathrm{C}$. PMT impulses were sent to a broadband $(0-200 \mathrm{MHz})$ preamplifier and then to a USB computer board, which was launched by additional electric pulses synchronous with the pulses of the LED. The signal of the board was processed by a personal computer with the Parsec (Dubna, Russia) software. As a result, the time interval between pulses was divided into 256, 512, or 1024 channels, and the computer showed the number of PMT impulses accumulated in each channel during the irradiation time, thus forming the kinetic curves of singlet oxygen phosphorescence after LED pulses.

The phosphorescence method provides more information on singlet oxygen than the trapping method. However, the trapping method is much more sensitive. Acetone was employed as the solvent for the singlet oxygen measurements because ETPA is readily soluble in it. The absorption spectrum of ETPA in acetone is shown in Supplementary Figure S3.

\subsection{Mouse Experimentation}

Mouse Experimentation was conducted in accordance with the ethical standards and recommendations for accommodation and care of laboratory animals covered by the Council Directives of the European community 2010/63/EU on the use of animals for experimental studies. The animal protocols were approved by the institutional animal ethics committee of A.N. Belozersky Research Institute of Physico-Chemical Biology, Approval Code: Protocol 8/21, Approval Date: 7 September 2021.

\subsection{Cell Culture and Intracranial Tumor Implantation}

C6 glioma cells were harvested with trypsin/versene while in the logarithmic phase of growth before intracranial stereotaxic implantation as a single cell suspension $\left(1 \times 10^{6}\right.$ cells $/$ $\mathrm{mL})$ into young C57BL/ 6 female mice $(18 \pm 3 \mathrm{~g})$. Under isoflurane anesthesia $(2-2.5 \%$ in air) by the SomnoSuite ${ }^{\circledR}$ system (Kent Scientific Corporation, Torrington, CT, USA), the mouse was placed in a stereotactic frame, and the skull was exposed through a midline incision cleared of connective tissue and dried. Implantation was performed at the following coordinates: $\mathrm{ML},-2.5 ; \mathrm{AP},-1.0 ; \mathrm{DV},-3.0$, as previously described [15]. C6 glioma cells $\left(5 \times 10^{5}\right.$ per mouse) were implanted with Robot Stereotaxic (Neurostar, Tubingen, Germany) using a Hamilton microsyringe at the speed of $3 \mu \mathrm{L} / \mathrm{min}$ in $10 \mu \mathrm{L}$ PBS.

\subsection{Glioma Photodynamic Therapy (PDT)}

PDT was performed 7 days after intracranial tumor implantation. Anatomical positioning of the tumor was obtained by brain magnetic resonance imaging (MRI) visualization. Tumor-bearing mice were sensitized via intravenous injection into the jugular vein of ETPA 
at $40 \mathrm{mg} / \mathrm{kg}$ of body weight $6 \mathrm{~h}$ pre-PDT; the drug dose regime was chosen following the study by [16]. Application of ETPA and PDT were conducted under isoflurane anesthesia. The brain skull in the illumination area was thinned with a milling cutter and illuminated for $30 \mathrm{~min}$ with a red laser light source $(\mathrm{L} 04-1 \mathrm{H}, 650 \mathrm{~nm}$, output power $100 \mathrm{~mW}$ ) producing a $1.5 \mathrm{~mm}$ diameter light beam, positioned on the region corresponding to the stereotaxic coordinates of the prior tumor injection. The surface of the skull was constantly cooled with saline to avoid thermal damage to the brain. Rectal temperature was kept constant at $37.0 \pm 0.2{ }^{\circ} \mathrm{C}$ using a heating pad. After PDT, the animals were returned to cages, provided with water and food ad libitum, and continually monitored for any signs of neurological deficit. The tumor-bearing group included 5 mice.

\subsection{Magnetic Resonance Imaging and Histological Studies of the Tumor Injury}

PDT-induced tumor injury was identified by analyzing brain MRI scans obtained 5 days after PDT on a 7-T magnet (Bruker BioSpec 70/30 USR; Bruker BioSpin, Ettlingen, Germany) using an $86 \mathrm{~mm}$ volume resonator for radiofrequency transmission and a phased array mice head surface coil for the reception. Before scanning, the animals were anesthetized with isoflurane $2-2.5 \%$ in a mixture of oxygen and air. Mice were placed in a prone position on a water-heated bed. The heads of the mice were immobilized using a nose mask and masking tape. The imaging protocol included a T2-weighted image sequence (time to repetition $=4500 \mathrm{~ms}$, time to echo $=12 \mathrm{~ms}$, slice thickness $=0.5 \mathrm{~mm}$ ). After MRI, the animals were sacrificed, and the brains were removed, fixed, sectioned, and stained by hematoxylin-eosin.

\section{Results}

\subsection{Chlorin Is Endogenous to O. sarsii}

Since Ophiuroidea as the class of the Echinodermata phylum were considered porphyrin-free [11], we hypothesized that the chlorin (3S,4S)-14-Ethyl-9-(hydroxymethyl)4,8,13,18-tetramethyl-20-oxo-3-phorbinepropanoic acid (ETPA) we previously discovered in the North Pacific Ophiura sarsii could result from the dietary consumption; the resulting seasonal variability in the ETPA content was also considered possible [10]. To address this issue, we performed a new $O$. sarsii collection in the same location but another season (December vs. May in [10]). Analytical HPLC (Figure 1A) of the butanol fraction of the ethanol extract of the freshly collected $O$. sarsii contained a major peak at a wavelength of $366 \mathrm{~nm}$ at the retention time of $52 \mathrm{~min}$, which corresponds to ETPA as the major $366 \mathrm{~nm}$ absorbing compound from our previous study [10], arguing against a seasonal diversity in the new chlorin compound in the brittle stars.

In order to directly rule out that ETPA could be a dietary derivative of $O$. sarsii, we separated the fresh catch of the brittle stars into two portions, one subjected to direct freezing and processing and the other fasted for 12 days prior to processing for preparative isolation (see Methods). Briefly, $15 \mathrm{mg}$ and $20 \mathrm{mg}$ of the butanol fraction of the EtOH extracts from non-fasted and fasted brittle stars, respectively, were subjected to semi-preparative chromatography (Figure 1B). Identical chromatograms were obtained for fasted and nonfasted preparations, with the major $366 \mathrm{~nm}$ absorption peak (retention time 34-37 min), corresponding to ETPA from our prior work [10], collected for subsequent analyses.

To control the identity of the compound in the collected fractions, spectrophotometry was performed in the wavelength range of $200-800 \mathrm{~nm}$. The resulting absorbance spectra were identical for the compound isolated from the fasted and non-fasted brittles stars and revealed the absorption typical for chlorins, with the absorbance peaks at 204, 293, 409, and $663 \mathrm{~nm}$ (Figure 2A), also fully coinciding with ETPA isolated by us from O. sarsii previously [10]. 

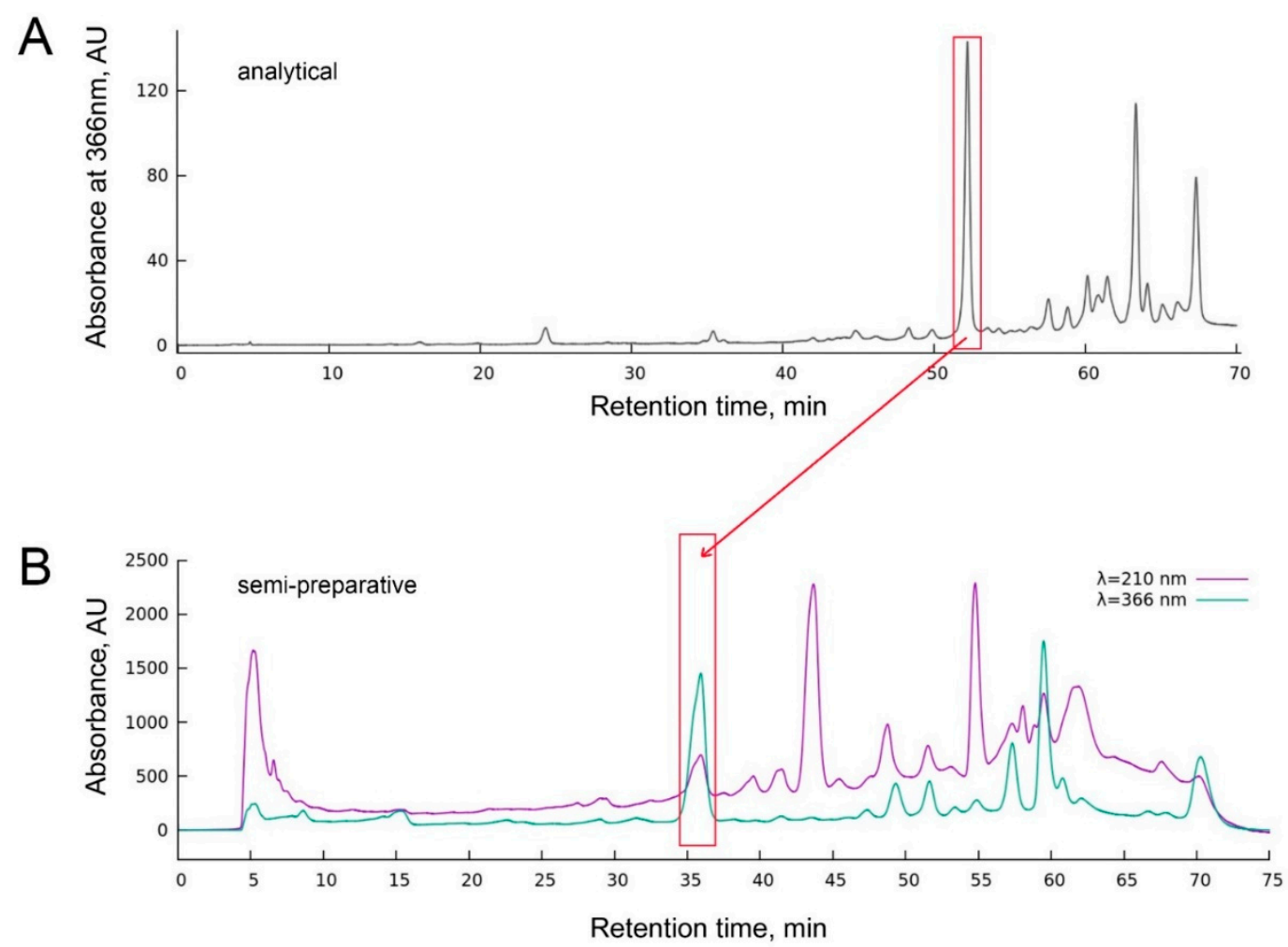

Figure 1. Analytical (A) and semi-preparative (B) chromatography of the butanol fraction of the EtOH extract of $O$. sarsii, absorption intensity at the wavelength of $366 \mathrm{~nm}(\mathbf{A})$ and $210 \mathrm{~nm} / 366 \mathrm{~nm}$ (B). The major peak at the retention time of $52 \mathrm{~min}$ (A) and $35 \mathrm{~min}$ (B) corresponds to ETPA (chlorin) from our previous study [10]. Chromatograms from non-fasted ophiuras are shown in both panels; samples from the fasted ophiuras show identical chromatograms.
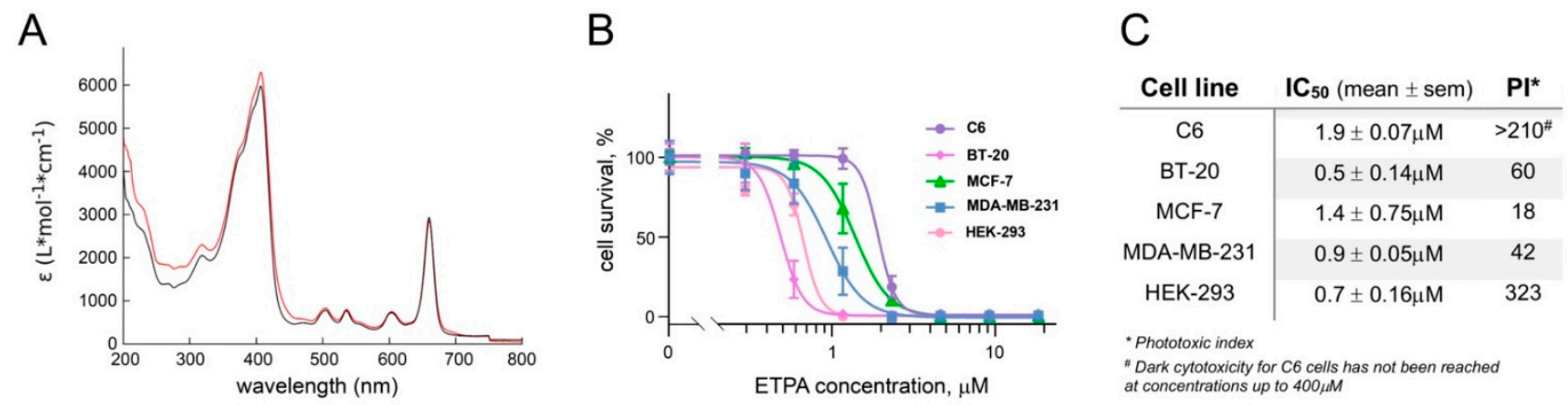

Figure 2. (A) Absorbance spectra of the chlorin compound isolated from the butanol fraction of the EtOH extract from control (red) and fasted (black) O. sarsii reveal absorbance peaks typical for chlorins, identical between the two preparations; (B) cell survival (MTT test) to evaluate the strong phototoxicity of ETPA after red-light irradiation. Data are given as mean \pm SEM, $\mathrm{n}=8$ (3 for HEK-293 cells); (C) ETPA phototoxicity $\mathrm{IC}_{50}$ and the phototoxicity index (PI, calculated as $\mathrm{IC}_{50}$ in the dark/ $\mathrm{IC}_{50}$ in the light [17]). Cell survival data in the dark are taken from [10] (breast cancer cell lines) or measured separately (C6 and HEK-293 cells, see Supplementary Figure S4). 
The final yield of the chlorin compound was $0.56 \mathrm{mg}$ from the non-fasted and $0.6 \mathrm{mg}$ from the fasted ophiuras, or $3.7 \%$ and $3 \%$, respectively. With these essentially identical yields, and with the lack of seasonal variability in the chlorin content, we concluded that ETPA is not part of the dietary preferences of the brittle stars but is endogenously synthesized by them.

\subsection{Phototoxicity of ETPA against a Panel of Cancer Lines}

The chlorin (ETPA) isolated from $O$. sarsii has shown dark cytotoxicity against a panel of breast cancer cell lines, with $\mathrm{IC}_{50} \mathrm{~s}$ in the range of 25-45 $\mu \mathrm{M}$ [10]. In order to assess whether the anticancer effect could be increased upon illumination of the compound to grant potential applicability for PDT, we next studied the phototoxic effect of ETPA.

Breast cancer cells lines BT-20, MCF-7, and MDA-MB-231, along with the glioma cell line C6 and non-cancerous HEK-293 cells, were preincubated for $2 \mathrm{~h}$ with increasing concentrations of ETPA before irradiation with a red-light LED lamp (580 to $780 \mathrm{~nm}$ ) for $30 \mathrm{~min}$ (fluence $=16.04 \mathrm{~J} / \mathrm{cm}^{2}$ ). Cell growth in the subsequent $72 \mathrm{~h}$ was assessed with the MTT assay (see Methods). Resulting data (Figure 2B,C) show striking phototoxicity of ETPA, with the sub-micromolar to low-micromolar $\mathrm{IC}_{50} \mathrm{~s}$ and impressive phototoxic indices (PI, measured as $\mathrm{IC}_{50}$ in the dark $/ \mathrm{IC}_{50}$ in the light [17], Figure $2 \mathrm{C}$ ), arguing for the strong potential of this natural chlorin in PDT applications. Notably, dark phototoxicity for the glioma C6 cells was not achieved at the highest concentrations of ETPA tested (Figure 2C and Supplementary Figure S4).

\subsection{Singlet Oxygen Production by ETPA}

Two methods to measure singlet oxygen production by ETPA upon illumination were employed (see Methods). The first was based on the chemical trapping of singlet oxygen by 1,3-diphenylisobenzofuran (DPIBF) and bleaching of DPIBF at $414 \mathrm{~nm}$ upon ETPA irradiation. The ETPA excitation was produced by the monochromatic $660 \mathrm{~nm}$ red light corresponding to the ETPA absorption maximum, which is not absorbed by DPIBF (Figure 2A and Supplementary Figure S3). Figure 3A shows the time-dependent decay in DPIBF $414 \mathrm{~nm}$ absorbance upon red-light illumination in the presence of ETPA. When comparing the efficiency of ETPA with that of meso-tetraphenylporphyrin (TPP, Figure 3B) known to produce singlet oxygen with the quantum yield of $0.7[12,13]$, the absolute quantum yield of singlet oxygen generation by ETPA was determined as 0.83 . Taking into consideration the relative error for such measurements at $\pm 10 \%$ of the average value, the singlet oxygen yield for ETPA can be estimated as $0.8 \pm 0.1$ from the trapping experiment.

The second method was based on detection of the infrared phosphorescence of singlet oxygen (see Methods), comparing the phosphorescence intensities (Figure 3C) in solutions of ETPA and phenalenone-one of the most efficient photosensitizers of singlet oxygen generation, with the quantum yield of this process close to one [14]. For calculations, so-called zero-time intensities $\left(\mathrm{I}_{\mathrm{o}}\right)$ of phosphorescence were used, which were obtained by extrapolation of the semilogarithmic kinetic plots to the zero time. The obtained $\mathrm{I}_{\mathrm{o}}$ values were then normalized to the absorption coefficients $\left(1-10^{-\mathrm{A}}\right)$ of the pigments at the wavelength of excitation. The resulting data are summarized in Figure 3D.

Thus, both methods indicate that the quantum yield of singlet oxygen production by ETPA is close to 0.8 , identifying ETPA as very a strong photosensitizer of singlet oxygen generation with promising for biomedical applications. 

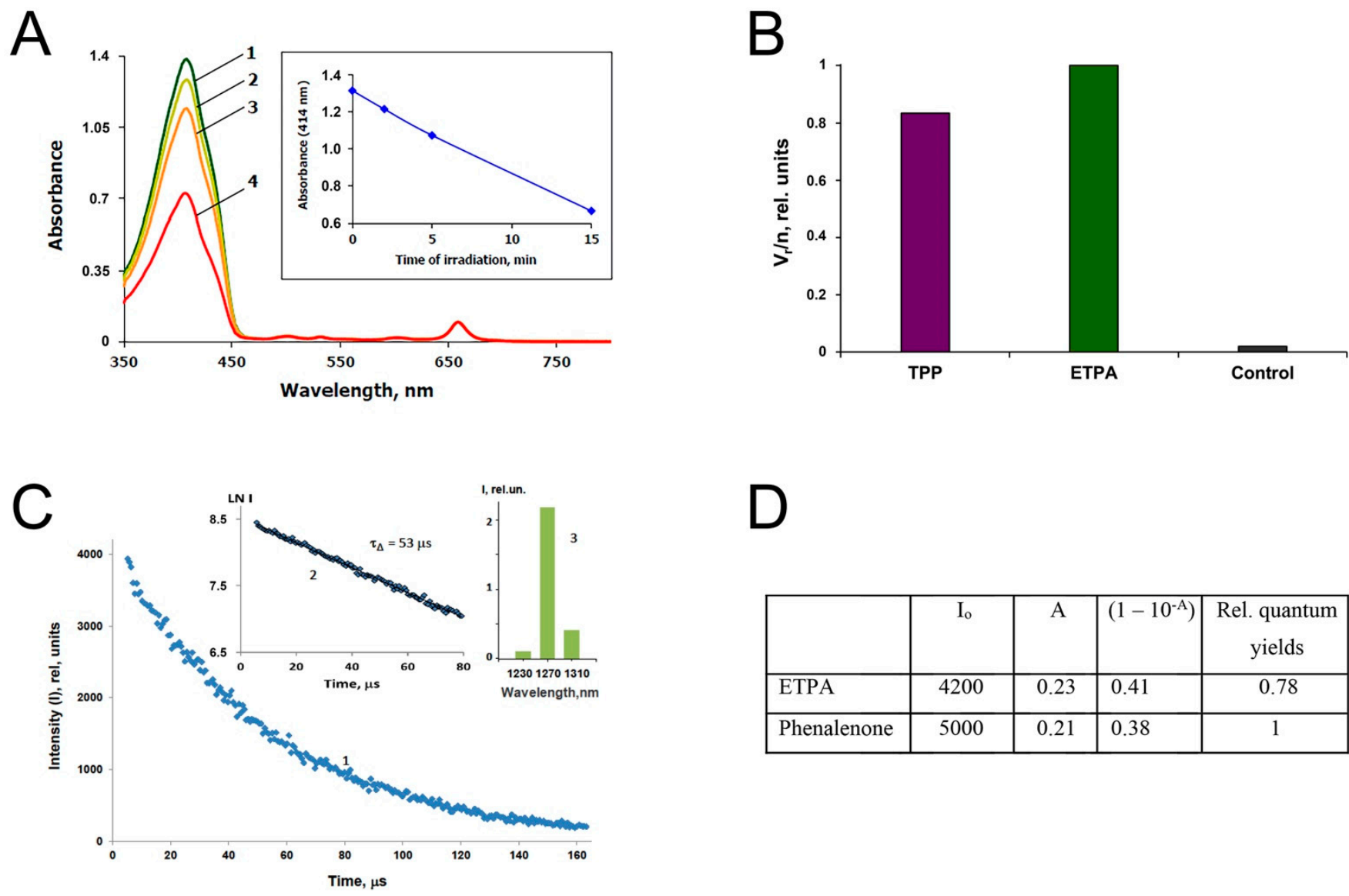

$\mathrm{D}$

\begin{tabular}{|l|c|c|l|c|}
\hline & $\mathrm{I}_{\mathrm{o}}$ & $\mathrm{A}$ & $\left(1-10^{-\mathrm{A}}\right)$ & $\begin{array}{c}\text { Rel. quantum } \\
\text { yields }\end{array}$ \\
\hline ETPA & 4200 & 0.23 & 0.41 & 0.78 \\
\hline Phenalenone & 5000 & 0.21 & 0.38 & 1 \\
\hline
\end{tabular}

Figure 3. ETPA efficiently generates singlet oxygen: (A) changes in the absorption spectrum of DPIBF (curves 1-4) in the mixture of DPIBF with ETPA in acetone during irradiation by monochromatic red light $(660 \mathrm{~nm})$ absorbed by ETPA. Here, "2" corresponds to $2 \mathrm{~min}$, " 3 " refers to $5 \mathrm{~min}$, and " 4 " to $15 \mathrm{~min}$ irradiation. Power of exciting light was $83 \mu \mathrm{W}$. Inset shows the time course of $414 \mathrm{~nm}$ absorption fall of DPIBF. ETPA bleaching was not observed; (B) the relative quantum yields of DPIBF oxidation $\left(\mathrm{V}_{\mathrm{r}} / \mathrm{n}\right)$ upon irradiation of TPP and ETPA. The rate of spontaneous DPIBF bleaching in the dark without sensitizer and irradiation is defined as "Control". For TPP, irradiation time was $10 \mathrm{~min}$, excitation wavelength was $512 \mathrm{~nm}$, irradiation power was $105 \mu \mathrm{W}$, and absorbance of TPP at $512 \mathrm{~nm}$ was 0.024 . For ETPA, irradiation time was $2 \mathrm{~min}$, excitation wavelength was $660 \mathrm{~nm}$, excitation power was $83 \mu \mathrm{W}$, and absorbance at $660 \mathrm{~nm}$ was $0.095 ;(\mathrm{C})$ kinetic trace of photosensitized phosphorescence of singlet oxygen upon excitation of ETPA by $5 \mu$ s pulses of violet LED (399 nm) in cartesian (1) and semilogarithmic (2) coordinates. Pulse repetition rate was $5 \mathrm{kHz}$, average LED power was $30 \mathrm{~mW}$, and irradiation (averaging) time was $10 \mathrm{~min}$. The PMT signal was accumulated using a time-resolved computer photon counting. The duration of one channel was $640 \mathrm{~ns}$, and the number of channels was 256. Absorbance of the solution at $396 \mathrm{~nm}$ was 0.246 in a $1 \mathrm{~cm}$ quartz cell. Here, " 3 " indicates phosphorescence emission spectrum estimated using three interchangeable interference filters. I corresponds to the phosphorescence intensity just after the end ( $5 \mu \mathrm{s})$ of the LED pulse. The decay time $\left(\tau_{\Delta}\right)$ of the phosphorescence exactly coincided with the known value of singlet oxygen lifetime in acetone; (D) calculation of the quantum yield of singlet oxygen by ETPA in comparison with phenalenone.

\subsection{Photodynamic Therapy with ETPA in a Mouse Model of Brain Tumor}

Inspired by the strong phototoxicity of ETPA against a panel of cancer cell lines in vitro, and by the especially high phototoxic index of our compound against glioma cells (Figure 2C), we next aimed at performing in vivo experiments of anticancer PDT with the brittle star-derived chlorin compound. Glioblastoma is the most common form of brain tumor, characterized by low responsiveness to treatment and poor prognosis (median 
survival < 2 years) [18]. Rat glioma C6 cells simulate human glioblastoma when injected into rats [19], and mouse brains [20,21] and have been a popular model in glioblastoma research [22]. We implanted C6 glioma cells in mouse brains (see Methods). After 7 days, IV administration of ETPA was performed, $6 \mathrm{~h}$ prior to brain MRI-guided targeted tumor illumination with a red laser $(650 \mathrm{~nm}$, see Methods and Figure 4A).
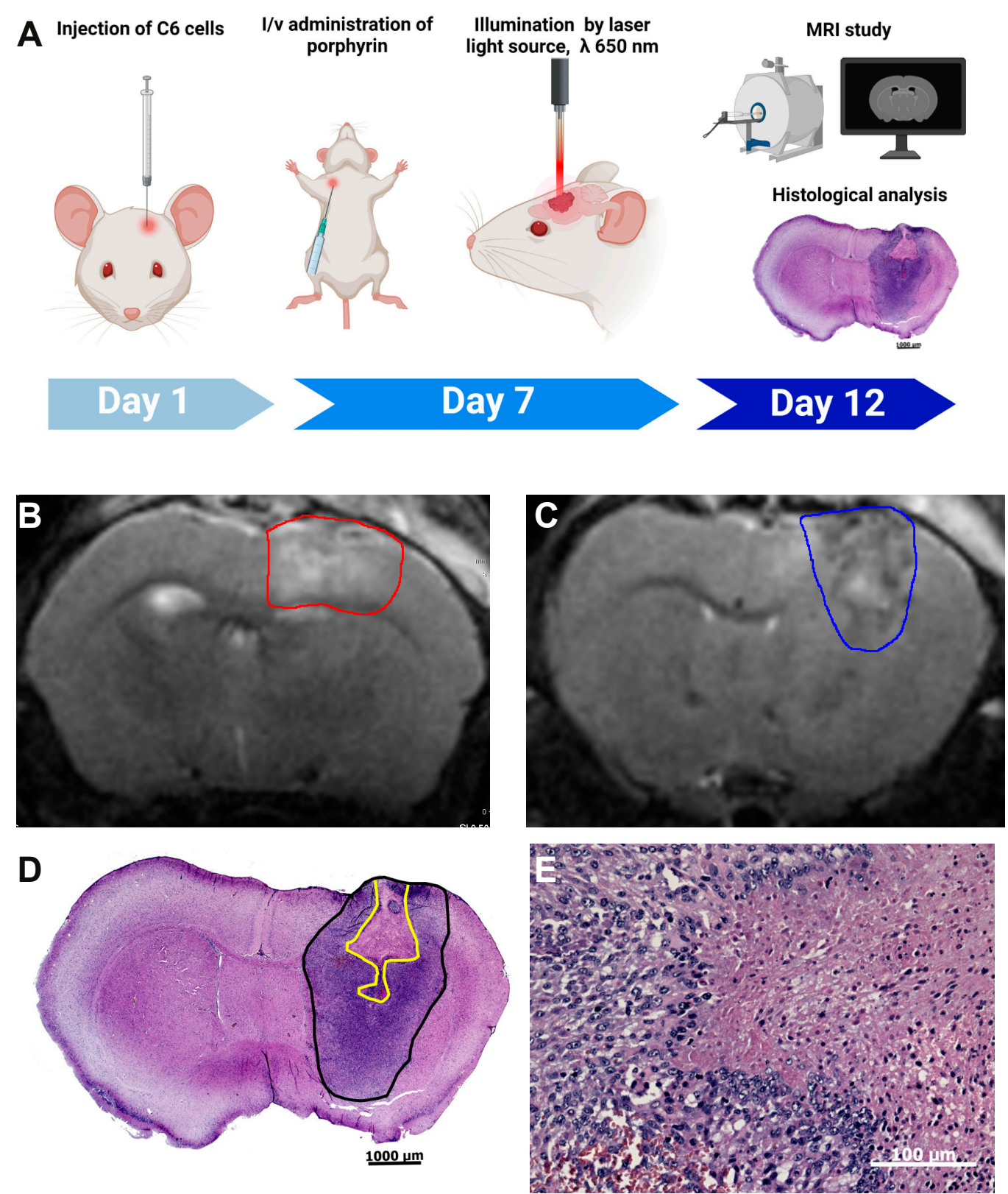

Figure 4. PDT using ETPA in a mouse model of glioblastoma: (A) scheme of the experiment; $(B, C)$ representative T2-weighted MR images from coronal brain sections ( $0.5 \mathrm{~mm}$ thick) obtained 5 days after PDT. The area outlined with a red line refers to hyperintensities regions (edema, B). The area outlined with a blue line refers to PDT-induced glioma necrosis (C); (D) a representative histological section, stained with eosin-hematoxylin, demonstrates the tumor boundaries (outlined in black) and the presence of necrotic loci in the area of laser illumination (outlined in yellow); (E) enlarged area showing the boundaries of necrotic and intact tumor tissues. Images shown are representative of 3 animals. 
Five days post PDT (Figure 4A), brain anatomical and histological analyses were performed. Through brain MRI, analysis of T2W-images revealed a strong signal change in the area exposed to laser irradiation. Figure 4B illustrates that in the tumor area exposed to laser irradiation, a hyperintense signal prevails indicating accumulation of water molecules in this area, i.e., tissue edema as the result of the photodynamic tissue damage. In the central part of the tumor (Figure 4C), the signal is mixed with zones of the hyperintense signal. Further histological analysis confirmed that this area corresponds to the area of necrosis (Figure 4D). Most of one hemisphere is occupied by a tumor consisting of poorly differentiated fusiform cells with large nuclei and narrow cytoplasm; many mitotic cells can also be seen. The stroma is poorly expressed. In the center of the tumor, there is an area of necrosis (a hypereosinophilic region devoid of nuclei), in the form of a pyramid with a broken apex. The area of necrosis occupies approximately $15 \%$ of the area of the tumor tissue in the section. At the border between dead and living tissue (Figure 4E), there are no reactive phenomena such as inflammation or connective tissue proliferation, and only blood vessels' dilatation can be noticed, along with numerous shapes (karyorrhexis, karyopycnosis, apoptotic bodies) of apoptotic cells. Notably, sham-operated and laserirradiated mice reveal no noticeable brain damage ([23]; data not shown).

Our findings are consistent with prior studies demonstrating the high efficiency of PDT for C6 glioma in vivo, with glioma damage observed in deep brain regions [16]. A synthetic porphyrin compound, 2,4-(a43-dihydroxyethyl)deuteroporphyrin IX tetrakiscarborane carboxylate ester (BOPP), was used in this study $[16,20]$. Other porphyrin-based photosensitizers (e.g., Photofrin) have been applied for PDT of brain tumors in rats [24] and in clinical studies [25]. Similarly, chlorin-based photosensitizers (Photolon, Talaporfin) have also been tested [26,27]. However, none has yet reached clinical approval for brain malignancies $[2,3,28]$.

A single dose (40 mg ETPA/ kg mouse body weight) was used in our study. This drug dose regime in our mouse model was chosen following prior studies on PDT in rat models of brain tumors (keeping in mind the drug dose interspecies conversion rate [29]). The dose consisted of $2.5 \mathrm{mg} / \mathrm{kg}$ for Photolon [26], $25 \mathrm{mg} / \mathrm{kg}$ for BOPP [16], $40 \mathrm{mg} / \mathrm{kg}$ for hematoporphyrin derivative [30], or $100 \mathrm{mg} / \mathrm{kg}$ for 5-aminolevulinic acid [31]. The purpose of our work was not to find optimal concentrations/conditions for PDT but rather to provide a proof-of-concept demonstration of the potential of a natural chlorin-ETPA from $O$. sarsii - to provide photodynamic damage to the brain tumor. It is worth noting that we chose a low illumination regime (10 times less, as compared with [16]) to avoid thermal damage to the brain. Despite this sparing regime, we managed to achieve necrotic death within the irradiated area, indicating the high potential of ETPA for PDT.

\section{Discussion}

Porphyrin derivatives as unique molecules showing powerful phototherapeutic effects have found applications in anticancer PDT [4]. In our previous work, a new natural chlorin from the Pacific brittle star Ophiura sarsii was discovered [10]. In this current work, we uncovered multiple novel elements to the biology of this compound, (3S,4S)-14-Ethyl-9(hydroxymethyl)-4,8,13,18-tetramethyl-20-oxo-3-phorbinepropanoic acid (ETPA) and its development toward PDT applications.

Although known in some marine invertebrates including some Echinodermata such as the sea urchin Strongylocentrotus purpuratus [8], porphyrins were considered absent in the class of Ophiuroidea [11]. Thus, following our discovery of ETPA in Ophiura sarsii, we hypothesized that this chlorin compound could be derived from a food source-perhaps a seasonal one- of these brittle stars [10]. However, our current data unequivocally demonstrate that ETPA is endogenous to this marine invertebrate. Subsequent research might be directed to the delineation of the biosynthetic routes of this first-ever Ophiuroidea porphyrin. Porphyrin biosynthesis has been well studied, with one of the conserved enzymes, aminolevulinic acid (ALA) synthase, producing the key intermediate 5-aminolevulinic acid from glycine and succinyl-CoA [8]. The Strongylocentrotus purpuratus genome encodes an 
ALA synthase $62 \%$ identical to the mouse ortholog $[8,32]$. ALA synthase has been cloned from other marine invertebrates such as the bivalve Patinopecten yessoensis, and conserved gene sequences have been identified across taxonomic groups [33]. It is thus conceivable that despite the lack of the brittle star O. sarsii genomic data, its ALA synthase could be cloned in the future, along with the genes encoding other components of the porphyrin biosynthetic route. Further investigations could also be directed to the question of the role ETPA plays in the brittle star. As a major metabolite that can be isolated in large quantities, this chlorin compound is likely to play an important biological role in $O$. sarsii, such as participation in the electron transfer processes or protection from predators.

To investigate the medical utility of ETPA, we here performed proof-of-concept PDT studies in vitro and in vivo. The former assessed the phototoxic properties of ETPA against a panel of breast cancer and glioma cell lines, revealing sub-micromolar efficiency upon redlight irradiation. The latter relied on a popular animal model of glioblastoma demonstrating a remarkable PDT effect. Six hours post IV injection of ETPA, targeted red laser irradiation produced a dramatic photoablation in the brain tumor, leading to glioma necrosis. Notably, in the course of our experimentation, we did not observe any acute toxicity in the mice due to IV injection of ETPA, agreeing with studies with other photosensitizers on the good tolerability (and brain barrier permeability) of this group of compounds (e.g., [16,20]). More detailed pharmacokinetics and pharmacodynamics studies will be performed in the future, along with the broader assessment of the laser irradiation protocols, to further optimize the applicability of ETPA for the treatment of brain tumors (and other tumors) in animal models. A modification of the method can also be conceivable using fiber optic implantation into deeper brain regions to achieve maximal and maximally focused irradiation in the desired area [34]. Such future developments could be promising given the fact that none of the currently available photosensitizers has yet been approved for brain tumor PDT [2,3] despite several reaching clinical studies $[25,27,28]$.

The abundance of ETPA in O. sarsii and the ease of its purification, along with its promising applications in PDT, make it attractive to consider upscaling of production of this natural product. Its abundance in its host is multiplied by the abundance of this brittle star species, wide-spread from Northern Atlantic, over the Arctic, and all the way to Northern Pacific, inhabiting waters from shallow to deep [35,36]. These features prompt considering mariculture of $O$. sarsii as has proven successful for other North Pacific invertebrates [37]. These possibilities, along with the preclinical developments of ETPA for PDT applications, may lead to the emergence of natural product-based novel photodynamic therapeutics.

Supplementary Materials: The following are available online at https://www.mdpi.com/article/ 10.3390/biomedicines10010134/s1, Supplementary Figure S1: Mechanism of photosensitized DPIBF oxygenation, Supplementary Figure S2: Mechanism of photosensitized IR phosphorescence of singlet oxygen, Supplementary Figure S3: Absorption spectrum of ETPA in acetone, Supplementary Figure S4: Dark cytotoxicity of ETPA against C6 and HEK-293 cells.

Author Contributions: Conceptualization, V.L.K. and Y.S.K.; methodology, M.B., V.A.B., A.S.B., A.S.K. and A.A.K.; validation, A.K., E.E.R. and D.S.; formal analysis, A.K., D.S. and A.A.K.; investigation, A.K., E.E.R. and D.S.; writing-original draft preparation, A.K., D.S., A.A.K. and V.L.K.; writingreview and editing, V.L.K.; supervision, A.A.K., Y.S.K. and V.L.K.; project administration, Y.S.K.; funding acquisition, V.L.K. and Y.S.K. All authors have read and agreed to the published version of the manuscript.

Funding: This research was funded by the Ministry of Science and Higher Education of Russian Federation, grant 13.1902.21.0012 "Fundamental problems of investigation and preservation of deep-sea ecosystems of potentially ore-bearing regions of the Northwestern Pacific" (Contract No. 075-15-2020-796). The researchers of the Federal Research Center (FRC) of Biotechnology were supported within the framework of the FRC State assignment.

Institutional Review Board Statement: The animal protocols were approved by the institutional animal ethics committee of A.N. Belozersky Research Institute of Physico-Chemical Biology, Approval Code: Protocol 8/21, Approval Date: 7 September 2021. 
Informed Consent Statement: Not applicable.

Data Availability Statement: The data presented in this study are fully available in the main text and supplementary materials of this article.

Acknowledgments: Figure 4A was created with BioRender.com. Accessed on 29 December 2021. Agreement number: HR23DJH3U2. We thank A. Begun for assisting in the fluence measurement.

Conflicts of Interest: The authors declare no conflict of interest.

\section{References}

1. Dougherty, T.J. Introduction. In Methods in Molecular Biology; Springer Science + Business Media: Berlin/Heidelberg, Germany, 2010; Volume 635, pp. 1-6. [CrossRef]

2. Li, X.; Lovell, J.F.; Yoon, J.; Chen, X. Clinical development and potential of photothermal and photodynamic therapies for cancer. Nat. Rev. Clin. Oncol. 2020, 17, 657-674. [CrossRef]

3. Baskaran, R.; Lee, J.; Yang, S.G. Clinical development of photodynamic agents and therapeutic applications. Biomater. Res. 2018, 22, 25. [CrossRef] [PubMed]

4. $\quad$ Pandey, R.K.; Goswami, L.N.; Chen, Y.; Gryshuk, A.; Missert, J.R.; Oseroff, A.; Dougherty, T.J. Nature: A rich source for developing multifunctional agents. Tumor-imaging and photodynamic therapy. Lasers Surg. Med. 2006, 38, 445-467. [CrossRef]

5. Cragg, G.M.; Newman, D.J. Nature: A vital source of leads for anticancer drug development. Phytochem. Rev. 2009, 8, 313-331. [CrossRef]

6. Katanaev, V.L.; Blagodatski, A.; Xu, J.; Khotimchenko, Y.; Koval, A. Mining Natural Compounds to Target WNT Signaling: Land and Sea Tales. In Handbook of Experimental Pharmacology; Springer: Cham, Switzerland, 2021; Volume 269, pp. 215-248. [CrossRef]

7. Katanaev, V.L.; Di Falco, S.; Khotimchenko, Y. The Anticancer Drug Discovery Potential of Marine Invertebrates from Russian Pacific. Mar. Drugs 2019, 17, 474. [CrossRef]

8. Calestani, C.; Wessel, G.M. These Colors Don't Run: Regulation of Pigment-Biosynthesis in Echinoderms. In Results and Problems in Cell Differentiation; Springer: Cham, Switzerland, 2018; Volume 65, pp. 515-525. [CrossRef]

9. Blagodatski, A.; Cherepanov, V.; Koval, A.; Kharlamenko, V.I.; Khotimchenko, Y.S.; Katanaev, V.L. High-throughput targeted screening in triple-negative breast cancer cells identifies Wnt-inhibiting activities in Pacific brittle stars. Sci. Rep. 2017, 7, 11964. [CrossRef] [PubMed]

10. Klimenko, A.; Huber, R.; Marcourt, L.; Chardonnens, E.; Koval, A.; Khotimchenko, Y.S.; Ferreira Queiroz, E.; Wolfender, J.L.; Katanaev, V.L. A Cytotoxic Porphyrin from North Pacific Brittle Star Ophiura sarsii. Mar. Drugs 2021, 19, 11. [CrossRef]

11. Kennedy, G.Y. Porphyrins in invertebrates. Ann. N. Y. Acad. Sci. 1975, 244, 662-673. [CrossRef] [PubMed]

12. Krasnovsky, A.A., Jr.; Kozlov, A.S.; Roumbal, Y.V. Photochemical investigation of the IR absorption bands of molecular oxygen in organic and aqueous environment. Photochem. Photobiol. Sci. 2012, 11, 988-997. [CrossRef]

13. Krasnovsky, A.A.; Kozlov, A.S. Photonics of dissolved oxygen molecules. Comparison of the rates of direct and photosensitized excitation of oxygen and reevaluation of the oxygen absorption coefficients. J. Photochem. Photobiol. A Chem. 2016, 329, 167-174. [CrossRef]

14. Krasnovsky, A.A.; Benditkis, A.S.; Kozlov, A.S. Kinetic Measurements of Singlet Oxygen Phosphorescence in Hydrogen-Free Solvents by Time-Resolved Photon Counting. Biochemistry 2019, 84, 153-163. [CrossRef] [PubMed]

15. Irtenkauf, S.M.; Sobiechowski, S.; Hasselbach, L.A.; Nelson, K.K.; Transou, A.D.; Carlton, E.T.; Mikkelsen, T.; deCarvalho, A.C. Optimization of Glioblastoma Mouse Orthotopic Xenograft Models for Translational Research. Comp. Med. 2017, 67, 300-314.

16. Hill, J.S.; Kahl, S.B.; Stylli, S.S.; Nakamura, Y.; Koo, M.S.; Kaye, A.H. Selective tumor kill of cerebral glioma by photodynamic therapy using a boronated porphyrin photosensitizer. Proc. Natl. Acad. Sci. USA 1995, 92, 12126-12130. [CrossRef]

17. Padrutt, R.; Babu, V.; Klingler, S.; Kalt, M.; Schumer, F.; Anania, M.I.; Schneider, L.; Spingler, B. Highly Phototoxic TransplatinModified Distyryl-BODIPY Photosensitizers for Photodynamic Therapy. ChemMedChem 2021, 16, 694-701. [CrossRef]

18. Tan, A.C.; Ashley, D.M.; Lopez, G.Y.; Malinzak, M.; Friedman, H.S.; Khasraw, M. Management of glioblastoma: State of the art and future directions. CA Cancer J. Clin. 2020, 70, 299-312. [CrossRef] [PubMed]

19. Auer, R.N.; Del Maestro, R.F.; Anderson, R. A simple and reproducible experimental in vivo glioma model. Can. J. Neurol. Sci. 1981, 8, 325-331. [CrossRef]

20. Hill, J.S.; Kahl, S.B.; Kaye, A.H.; Stylli, S.S.; Koo, M.S.; Gonzales, M.F.; Vardaxis, N.J.; Johnson, C.I. Selective tumor uptake of a boronated porphyrin in an animal model of cerebral glioma. Proc. Natl. Acad. Sci. USA 1992, 89, 1785-1789. [CrossRef] [PubMed]

21. Fan, Y.; Cui, Y.; Hao, W.; Chen, M.; Liu, Q.; Wang, Y.; Yang, M.; Li, Z.; Gong, W.; Song, S.; et al. Carrier-free highly drug-loaded biomimetic nanosuspensions encapsulated by cancer cell membrane based on homology and active targeting for the treatment of glioma. Bioact. Mater. 2021, 6, 4402-4414. [CrossRef]

22. Grobben, B.; De Deyn, P.P.; Slegers, H. Rat C6 glioma as experimental model system for the study of glioblastoma growth and invasion. Cell Tissue Res. 2002, 310, 257-270. [CrossRef]

23. Romanova, G.A.; Silachev, D.N.; Shakova, F.M.; Kvashennikova, Y.N.; Viktorov, I.V.; Shram, S.I.; Myasoedov, N.F. Neuroprotective and antiamnesic effects of Semax during experimental ischemic infarction of the cerebral cortex. Bull. Exp. Biol. Med. 2006, 142, 663-666. [CrossRef] 
24. Zhang, X.; Jiang, F.; Kalkanis, S.N.; Yang, H.; Zhang, Z.; Katakowski, M.; Hong, X.; Zheng, X.; Chopp, M. Combination of surgical resection and photodynamic therapy of 9L gliosarcoma in the nude rat. Photochem. Photobiol. 2006, 82, 1704-1711. [CrossRef]

25. Aziz, F.; Telara, S.; Moseley, H.; Goodman, C.; Manthri, P.; Eljamel, M.S. Photodynamic therapy adjuvant to surgery in metastatic carcinoma in brain. Photodiagnosis Photodyn. Ther. 2009, 6, 227-230. [CrossRef] [PubMed]

26. Tzerkovsky, D.A.; Osharin, V.V.; Istomin, Y.P.; Alexandrova, E.N.; Vozmitel, M.A. Fluorescent diagnosis and photodynamic therapy for C6 glioma in combination with antiangiogenic therapy in subcutaneous and intracranial tumor models. Exp. Oncol. 2014, 36, 85-89. [PubMed]

27. Akimoto, J.; Haraoka, J.; Aizawa, K. Preliminary clinical report on safety and efficacy of photodynamic therapy using talaporfin sodium for malignant gliomas. Photodiagnosis Photodyn. Ther. 2012, 9, 91-99. [CrossRef]

28. Quirk, B.J.; Brandal, G.; Donlon, S.; Vera, J.C.; Mang, T.S.; Foy, A.B.; Lew, S.M.; Girotti, A.W.; Jogal, S.; LaViolette, P.S.; et al. Photodynamic therapy (PDT) for malignant brain tumors-where do we stand? Photodiagnosis Photodyn. Ther. 2015, 12, 530-544. [CrossRef] [PubMed]

29. Nair, A.B.; Jacob, S. A simple practice guide for dose conversion between animals and human. J. Basic Clin. Pharm. 2016, 7, 27-31. [CrossRef] [PubMed]

30. Kaye, A.H.; Morstyn, G. Photoradiation therapy causing selective tumor kill in a rat glioma model. Neurosurgery 1987, $20,408-415$. [CrossRef]

31. Olzowy, B.; Hundt, C.S.; Stocker, S.; Bise, K.; Reulen, H.J.; Stummer, W. Photoirradiation therapy of experimental malignant glioma with 5-aminolevulinic acid. J. Neurosurg. 2002, 97, 970-976. [CrossRef]

32. Cameron, R.A.; Samanta, M.; Yuan, A.; He, D.; Davidson, E. SpBase: The sea urchin genome database and web site. Nucleic Acids Res. 2009, 37, D750-D754. [CrossRef]

33. Mao, J.; Zhang, Q.; Yuan, C.; Zhang, W.; Hu, L.; Wang, X.; Liu, M.; Han, B.; Ding, J.; Chang, Y. Genome-wide identification, characterisation and expression analysis of the ALAS gene in the Yesso scallop (Patinopecten yessoensis) with different shell colours. Gene 2020, 757, 144925. [CrossRef]

34. Cho, J.; Kwon, D.H.; Kim, R.G.; Song, H.; Rosa-Neto, P.; Lee, M.C.; Kim, H.I. Remodeling of Neuronal Circuits After Reach Training in Chronic Capsular Stroke. Neurorehabilit. Neural Repair 2016, 30, 941-950. [CrossRef] [PubMed]

35. Lütken, C.F. Contribution to knowledge about the brittle stars. II. Overview of the West Indian ophiuras (title in Danish). Vidensk. Medd. Dan. Nat. Förening Kjøbenhavn 1856, 7, 1-19.

36. Harris, J.L.; MacIsaac, K.; Gilkinson, K.D.; Kenchington, E.L. Feeding biology of Ophiura sarsii Lutken, 1855 on Banquereau bank and the effects of fishing. Mar. Biol. 2009, 156, 1891-1902. [CrossRef]

37. Gavrilova, G.S.; Kucheryavenko, A.V. Commercial rearing of the sea cucumber Apostichopus japonicus in Peter the great bay: Methodical peculiarities and results of the work of a mariculture farm in Sukhodol Bight. Russ. J. Mar. Biol. 2010, 36, 539-547. [CrossRef] 